\title{
An Agent-based Simulator Applied to Teaching-learning Process to Predict Sociometric Indices in Higher Education
}

\author{
Iván García-Magariño, Inmaculada Plaza, Senior Member, IEEE, Raúl Igual, Member, IEEE, Andrés S. Lombas, \\ Hana Jamali
}

\begin{abstract}
Most novice teachers and even some experienced teachers can lack appropriate tools for designing teaching strategies that ensure the quality of education. The ability of working in teams is crucial in educating professionals. The literature proves that social relations influence the performance of teams. For instance, the team cohesion is directly related with its performance. In the current work, we have developed an agent-based tool for assisting teachers in simulating their teaching strategies to estimate their influence on the group sociometrics like cohesion, coherence of reciprocal relations, dissociation and density of relations. The experiments with nine scenarios in disciplines of computer science, electronic, psychology, business, tourism and renewal energies with 239 students and six teachers including experienced and novice ones show that its underlying agentbased framework can adapt to different disciplines obtaining similar outcomes to the real ones. We learned that the tool was especially reliable in predicting the density of relations and the cohesion, being the latter one probably the most relevant due to its known relation with academic performance. In addition, we also learned that it was difficult to assess the prediction quality of the dissociation in higher education, due to the usual low amounts or absence of reciprocal rejections in the students' groups in this educational stage. The presented agent-based tool is publicly distributed as open source for facilitating other researchers in following this research line.
\end{abstract}

Index Terms-agent-based simulator, agent-based social simulation, higher education, multi-agent system, sociogram

I. García-Magariño is with the Department of Software Engineering and Artificial Intelligence, Complutense University of Madrid, Madrid, 28040 Spain e-mail: igarciam@ucm.es (see http://grasia.fdi.ucm.es/ivan/).

I. Plaza is with the Department of Electronic Engineering and Communication, University of Zaragoza, E.U. Politécnica de Teruel and Instituto de Investigación Sanitaria Aragón, Teruel, 44003 Spain e-mail: inmap@unizar.es

R. Igual is with the Department of Electric Engineering, University of Zaragoza, E.U. Politécnica de Teruel, and Instituto de Investigación Sanitaria Aragón, Teruel, 44003 Spain e-mail: rigual@unizar.es

A. S. Lombas is with the Department of Psychology and Sociology, University of Zaragoza, Facultad de Ciencias Sociales y Humanas de Teruel, Teruel, 44003 Spain e-mail slombas@unizar.es

H. Jamali is with the Superior School of Technology, Cadi Ayyad University, Essaouira, 44000 Morocco e-mail jamali.hanaa@gmail.com

NOTICE: This is the authors' accepted manuscript version of a work that was published in the IEE Transactions on Learning Technologies journal. This document does not include the publisher value-added contributions such as copy-editing, formatting, technical enhancements and (if relevant) pagination. A definitive version was subsequently published in this journal with DOI: http://dx.doi.org/10.1109/TLT.2019.2910067

PLEASE CITE AS:

García-Magariño, I. Plaza, I., Igual, R., Lombas, A.S., \& Jamali, H. (2019). An agent-based simulator applied to teaching-learning process to predict sociometric indices in higher education. IEEE Transactions on Learning Technologies, http://dx.doi.org/10.1109/TLT.2019.2910067

\section{INTRODUCTION}

\section{A. The Use of Simulations in Education}

Q IMULATION software is becoming an important tool in education that has been applied in different domains. For instance, simulation has shown to be useful for increasing students' academic performance in nursing [1], [2], medicine [3], pharmacology [4], business [5] and economy [6]. In these studies, students used simulations to learn the effects of some actions and/or conditions. Simulation has also been used for training teachers. For example, simulations with virtual avatars have been used for active teaching training [7], and to effectively ask questions to children in suspicious circumstances of abuse and maltreatment [8]. Unlike the aforementioned works, where simulations have been used as technology for training people's specific abilities, our work has focused on predicting students' interactions in the classroom. The goal of our simulations is to support teachers' decisions in designing teaching strategies and distributing students in classrooms to improve students' interactions, so that teaching-learning process is favored.

\section{B. Sociometric Questionnaire}

Several questionnaires that evaluate students' interactions in the classroom, have been developed. The sociometric questionnaire of Moreno [9] is frequently used to measure social relationships. We have used this questionnaire in our studies [10]. This questionnaire collects information about the interaction between persons (acceptance or rejection emitted and received by an individual). Based on this information, social relationships can be quantified applying a method called sociometry. Sociometry uses indices of group structure (such as cohesion, dissociation, coherence and density) and sociograms (a graphic representation that shows the structure of interpersonal relations in a group). In our studies, to simulate sociometric indices and sociograms, we have developed agentbased simulators (ABSs) since ABSs have been considered to be useful for the simulation of social aspects [11].

\section{Sociometry in Education}

In educational setting, sociometry has been used to reveal (a) school group dynamics and (b) the structure and hierarchy of student groups. After analyzing these factors, one can determine the group leader, the marginalized individuals, 
group cohesion and status of each member in a group. This knowledge can help to organize groups for improving group cohesion and stimulating positive relationships, which can affect the evolution and results of the group. It may also help to solve problems of adaptation, integration and socialization of the students in a group. In this regard, Ioan et al. [12] and Jostad et al. [13] remarked the importance of sociograms in education and provided examples of the use of sociograms in educative activities, respectively in a sport context and an outdoor-adventure context. Based on data obtained from a football team of a school through the sociometric questionnaire, Ioan et al. [12] identified the leader of the team, the best favorite person, a group of players with mutual elections, a group of players with mutual rejections and the cohesion level of the team. Petracovschi et al. [14] analyzed the social integration of children with weight issues in a school using the sociometric questionnaire. Once she identified those children with low social integration, she organized exercises in physical-education lessons and extracurricular activities to reduce social rejection of these children.

Research on sociometric indices has shown that these are related to better psychosocial adjustment and academic achievement. Thus, in relation to psychosocial adjustment, popular students (i.e. those who are accepted by most peers, and have good interpersonal abilities and high levels of empathy) have the tendency to cooperate with others [15]. Moreover, peer acceptance is positively associated with social prominence [16]. On the contrary, rejected students are perceived as unpleasant and are less liked [17], and have more conflictual relationships with their peers and teachers, being more frequently involved in disruptive and aggressive behaviors that lead to the violation of institutional rules [18]. With regard to academic achievement, peer acceptance predicts academic achievement in children [19] and adolescents [20], [21].

From a social network perspective, learning constitutes a relational process that depends on and involves interactions (e.g., information exchanges, discussions, dialogic feedback and questioning) [22]. But, whereas in school education learning is mainly based in the dual interaction between the student and the teacher, in higher education the students and the teacher constitute a learning community that together builds a social learning experience. Another distinctive characteristic of higher education is that a great amount of interactions between students occurs in on-line learning environments. This is one of the main reasons why sociometric research analyzes social networks of learning communities in higher-education.

Zhao et al. [23] analyzed messages posted by students in discussion forums in an on-line learning environment through sociograms, and the results illustrated the characteristics of group interaction in the course. Sociograms were also used to evaluate teaching experiences aimed at improving conflict resolution and social values. Macfadyen and Dawson [24] found that student academic achievement could be predicted using the data obtained from typical learning management systems used in most higher education courses. They recommended to plot the data extracted from the forum discussion in a sociogram, since it allowed easily identifying high and low-performing students. Those students that established more connections with their peers tended to be high-performing students, whereas those students that established few connections tended to be low-performing students. However, this recommendation was not supported with statistical analysis. This was done later by Dawson in another study [25]. Additionally, she found that high-performers developed larger social networks than low-performers. Taking all the results together into account, they pointed out that sociograms may allow one to identify patterns of networks of high- and low-performing students based on their network size and composition.

\section{Simulations to Predict Sociometric Indices and Sociograms of Classes}

As mentioned earlier, our work has focused on performing simulations to predict sociometric indices and sociograms from a class. Thus, in a first study [10], we developed a tool called CLUS-SOCI (an agent-based and CLUStering tool for simulating SOCIograms) that was able to simulate sociometric indices and class sociograms based on psychological characteristics of students. With this tool, teachers could arrange classes in a way that conflictual relationships could be reduced and friendly relationships could be increased. Although the tool successfully simulated social relationships of students, the simulation only considered a partial aspect of teaching-learning process. In accordance with the model proposed by Dunkin and Biddle [26], in addition to studentsrelated variables, teacher-related variables are also involved in the teaching-learning process, and these two variable types determine the interactive behaviors of the teacher and students in the classroom, which produce different academic, social and emotional consequences. Dunkin and Biddle's model is not the only theoretical proposal that highlights the importance of relationships of students. In fact, there is a general agreement in theories and research that positive meaningful relationships with others are cornerstone of young people's capacity to function effectively in social, affective and academic domains. In particular in the academic domain, these relationships have proven to affect academic motivation, engagement and achievement (for a review, see [27]). Hence, it is well accepted that the degree to which students enjoy positive interactions with peers is especially important to understand their adaptation and ultimate achievement in school [28]. The reason of this is that accepted individuals experience more opportunities to learn adaptive modes of social behaviors, social cognitions and emotional support that dispose them towards better academic results. On the contrary, low-accepted individuals are less likely to have a positive social or academic reputation established in the peer group, which in turn causes them to be ignored and ridiculed, having less opportunities to experience interactions with peers [29].

With the purpose of designing a more complete simulation of the teaching-learning process, in a second study, we [30] developed a new ABS that incorporated teacher-related variables. More concretely, we introduced teaching strategies as a teacher-related variable. This study showed that the new ABS correctly predicted social relationships among students. 
However, our previous studies suffer from some limitations that are worth noting. This study [30] was only tested with data extracted from scientific literature with students of nurse and sport science degrees. And in another of our studies [31], the simulations were not assessed by comparing their predicted outcomes with real data. Bearing all this in mind, the present work sought to overcome these limitations. To do so, we developed a new ABS, named FTS-SOCI 2 (an advanced Framework for simulating Teaching Strategies with evolutions of SOCIograms), and evaluated statistically its predictive capacity to estimate social relationships among students with data collected by us. Moreover, in order to assess the generality of the simulator, we tested simulations with the new ABS in different disciplines: electronic and automation engineering, computer science engineering, psychology, business and management, tourism, and renewal energies.

\section{FTS-SOCI 2}

FTS-SOCI 2 is an ABS tool specifically designed and developed for supporting decisions of higher-education teachers regarding the planning of their teaching strategies. The source code is publicly available from a dataset of Mendeley research data repository ${ }^{1}$. In particular, this tool focuses on simulating the social relations of groups of students given a certain teaching strategy and a group of students classified in certain behavioral types. The teaching strategies are defined using a certain group of learning activity types. This group of activity types was designed based on the knowledge gathered from the literature and the expertise of a group of teachers in two different engineering grades and the psychology grade. FTSSOCI 2 allows simulating the influence of several teaching strategies in the same simulation timeline. Each teaching strategy can start at certain point and last for a certain duration. In this way, the simulator tool allows users to simulate both simultaneous and consecutive strategies.

Section II-A introduces the way of defining new teaching strategy with the framework of FTS-SOCI 2, introducing the supported kinds of learning activities and the way of determining the schedule of these. Section II-B presents the user interface of the tool.

\section{A. Definition of Teaching Strategies}

Firstly, the instructor must determine some basic features of the course in which the strategy is going to be applied. In particular, they must indicate the duration in terms of the number of lectures, considering that each lecture takes between $50 \mathrm{~min}$ and one hour. This duration is necessary so that the strategy can be applied in a limited interval time (e.g. a semester) within a longer academic period (e.g. the whole academic year). They must also determine the number of lectures per week, so that the simulator can properly interpret a request of performing an activity in a specific week or month.

\footnotetext{
${ }^{1}$ FTS-SOCI 2 source code for simulating teaching strategies to predict sociometric indices in higher education, Mendeley Data, v1 http://dx.doi.org/10.17632/n8b4wvvd74.1
}

In order to simulate the sociogram of students considering the teaching strategy, instructors must determine the collaborative activities of their teaching strategy. More concretely, the instructor must determine the following information for each activity:

- The activity type: The presented approach provides different types of activities based on the experience in several grades. The current framework supports the activity types mentioned later in this section.

- The position in the timeline of the strategy: This information refers to the chronological-order position of the lecture in which the activity begins. This position can be expressed either indicating the week or the month, so that instructor can easily define their strategy. This position only considers the lectures of the defined strategy, even if this strategy does not start at the beginning of the simulation. In this way, strategies can be defined without deciding when these are going to be applied in the global simulation.

- The activity duration: This duration determines the number of hours that each student should work. This time can be spent in classes and/or as homework activities.

- The size of teams when applicable: Most available collaborative activity types are performed by several teams in a class. The instructor should determine a recommended number of students for each team.

- The level of interaction: This approach associates a "high" level of interaction to a learning activity when the collaborative working time is estimated to be greater than the individual working time. If this is not the case, it associates a "low" level of interaction to the corresponding activity.

The set of learning activity types was determined considering different areas such as nursing, sport lessons, electronics and automation engineering, computer science engineering, and psychology. In particular, FTS-SOCI 2 supports the simulation of the following activity types: hardware assembly, software development, document writing, problem solving with tutoring representation, discussion and role-play. These activity types are introduced when presenting the questionnaire about the teaching strategy in section III-B3.

Notice that all the aforementioned learning activities are collaborative, since these are the ones with the highest impact on interactions among students. However, even non-collaborative activities may have impact on student interactions although this is much lower. In FTS-SOCI 2, all the non-collaborative activities are simulated in the same way regarding the number lectures, and consequently the simulator does not need to interpret executable code regarding which non-collaborative facilitation technique is applied in each lecture. In order to support the definition of complete teaching strategies, teachers can indicate the facilitation techniques as comments within the programming code. Since the programming comments are not executed, each teacher can indicate any non-collaborative facilitation technique that they find appropriate.

A teaching strategy is defined by simply creating a new class that inherits from the "Teacher Extended" class and 
implementing its constructor and its "Live" abstract method. The constructor must call its superclass constructor indicating respectively the duration of the strategy and the number of lectures per week in their parameters.

The Live method is invoked in every lecture. Since normally each activity is only performed in several months or weeks, the framework provides some methods to support this. More specifically, the methods "Is In Month" and "Is In Week" determine whether the current lecture is the first one of respectively a given month or a week specified in the parameter by its position. The "Get Month" and "Get Week" methods return (a) the position of the current period (respectively month or week) when the actual lecture is its first one, or (b) the "minus one" value otherwise. In this manner, the definition of the strategy can use control structures of the Java programming language to define some strategies with less programming statements. For example, one can determine the application of certain activities in certain weeks, by comparing the returned week values with certain week positions. As another example, one can determine activities every two weeks by means of the remainder operation over the returned week value.

The application of a particular activity is determined with the "Perform Activity" method. The first parameter is a value of the "Activity" enumeration with the activity types mentioned earlier in this section. The second parameter is the duration expressed as the number of lectures, and the third one is the level of interaction.

The conformation of teams or groups is performed with a different method called "Make Groups". This method receives input from a parameter with the preferred size of groups. It organizes the class in different teams, and the corresponding activities invoked later use these teams. This method is implemented as a different one from Perform Activity method, so that the same teams perform different activities as usual in real teaching strategies. In addition, this saves simulation time, since the procedure of organizing the teams is only invoked when it is really necessary. This method should be invoked before the first activity that is performed with teams. Figure 1 shows an example of a teaching strategy defined with FTSSOCI 2.

\section{B. User Interface}

Figure 2 shows the main frame of the user interface of FTSSOCI 2. One of its most relevant features is that it allows users to run simulations with several teaching strategies that can be simultaneous and/or consecutive. In particular, the user can add teaching strategies to the list of simulated strategies indicating the start point. Each strategy is defined for a given number of lectures, it starts in the lecture position defined in the interface, and goes on until it reaches the number of lectures of strategy. If the user wants to simulate consecutive courses, the duration of the simulation is the sum of the lectures of the courses, and the start point must be set so that, when the teaching strategy of one course finishes, the second strategy starts right after. If several courses are planned to be simulated simultaneously, then the duration of the simulation will be the duration of the longest strategy, and both strategies starts at the same point.
In addition, several combinations can be configured. In the example of the execution, the simulation is defined for an academic course, where two teaching strategies are conducted in two courses of the first semester, and another strategy is applied in a course of the second semester, conforming a oneyear simulation.

When the simulation is executed, the evolution of a sociogram is simulated. This evolution is shown with an animation in another frame, as one can observe in the execution example of Figure 3. The different types of students are represented with the color notation shown in the right-side legend. The selection and rejection relations are represented with different colors. Sociometric measurements are displayed in the right-bottom area.

\section{STUDY}

\section{A. Participants}

Six university teachers (four male, two female) from the University of Zaragoza in Spain and Cadi Ayyad University in Morocco participated in the current work. Two of these teachers were novice (one without any experience before and the other with only one year of part-time experience), one had medium experience (six years of experience), and the other three were experienced (each one with more than ten years of experience). Only one out of the six teachers had previous experience in participating in surveys related to sociometric before the presented experiment.

Participant students were taken from different undergraduate subjects, which were introduction to computers, physics and electronics, programming I, electrotechnics, research methodology, management techniques, tourism, renewable energies, and software engineering. There were a total of 239 students. $51.4 \%$ of the participants were male, and their average age was 20.3 years old. In particular, 96 of these participants were studying in the University of Zaragoza, and 143 participants were studying in the Cadi Ayyad University. Regarding the social background, the students of University of Zaragoza studied in the campus of Teruel city, which is a really small city of Spain with only about 35,500 inhabitants. A great part of these students were born in one of the nearest big cities, i.e. Zaragoza or Valencia, but needed to change their place of residence for different academic reasons. Cadi Ayyad University is one of the largest universities in Morocco, and theirs students lived in Marrakech city, which is much bigger that Teruel with about 1,500,000 inhabitants. The culture and religion backgrounds of students were different between these two universities, since one university belongs to an area with occidental European culture, whereas the other belongs to a North African Arab country. The selection process firstly selected the teachers that were willing to participate in the study, and these teachers selected their subjects without any specific criteria. They presented this study as part of the course, and dedicated time within lessons to complete it. Although responding was not strictly mandatory, the teachers highly encouraged their students to do it. In fact, the reason of the few students missing the surveys was mainly that they did not attend to the lessons in which the survey was filled. 


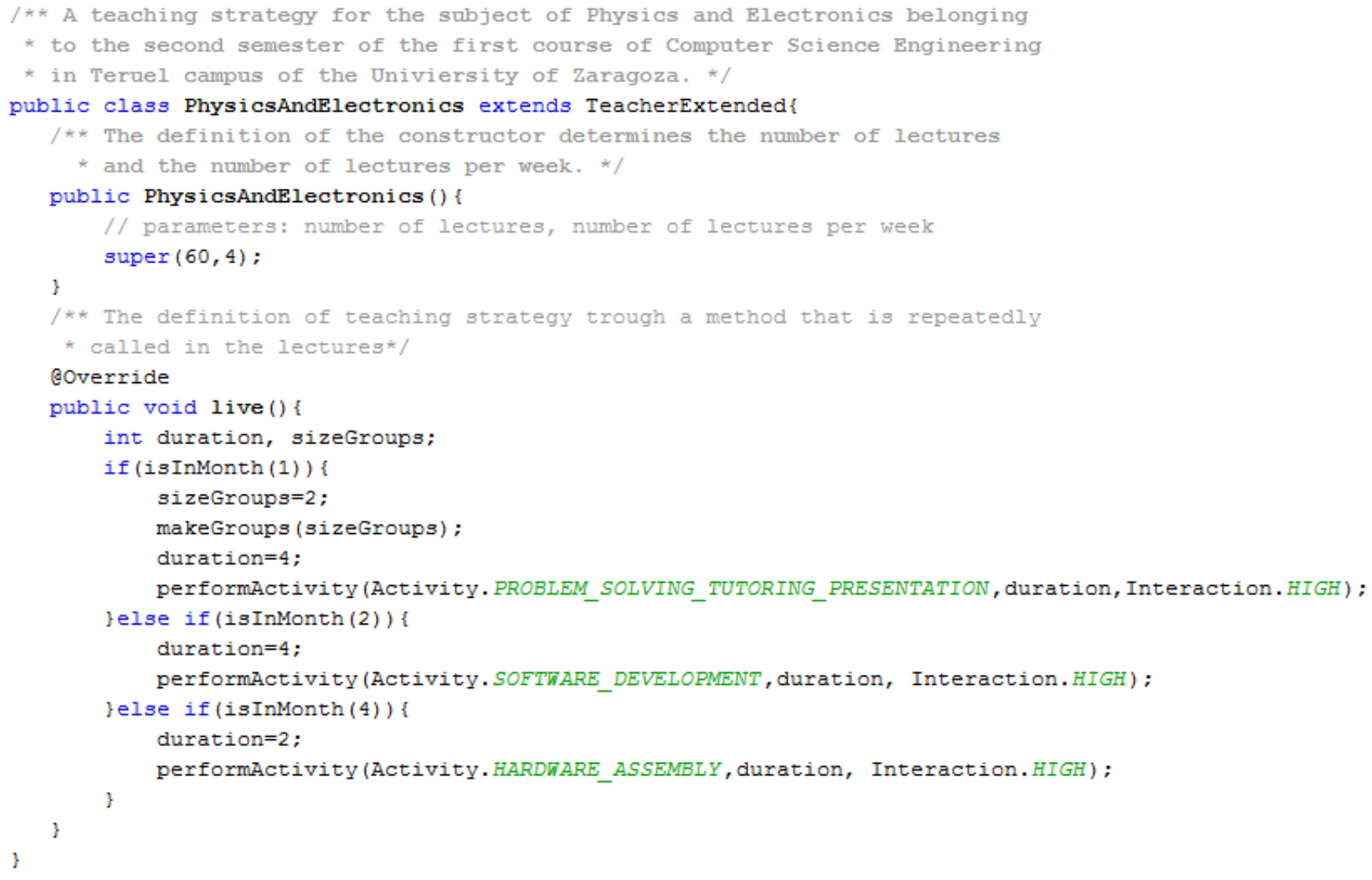

Fig. 1. An example of a teaching strategy definition

\section{B. Measures}

1) Sociometric Questionnaire: Students were given a list of the names of their classmates and were asked to respond to the following two questions: (1) "Who would you choose as workmate for learning activities?", and (2) "Who would you avoid as workmate for learning activities?" These items determined the selections and rejections among students, respectively. Based on the answers given by the participants on these questions, it is possible to measure the following group sociometric indices: Cohesion (the degree of mutual acceptance between members of a group; IAg), Dissociation (the degree of mutual rejections between members of a group; IDg), Coherence (the relation between reciprocal acceptance and the number of total acceptance selections received by the members of a group; ICg) and Group Intensity (the degree of acceptance and rejection selections received by the members of a group; IIg). These indices were calculated for each class, according to the definitions and formulas of these indices provided by Barrasa and Gil [32], which are further introduced in the Appendix. Based also on the answers given by the participants, one can create graphic representations (named sociograms), which plots the selections and rejections among students.

2) Classroom Behavior Questionnaire: Students were asked to classify their predominant behavior in the classroom in one of the following categories: (1) quiet students, (2) participant students, (3) tangent students (who lead discussions to tangent topics), (4) joker students (who tell classmates jokes), (5) obstructive students (whose behaviors are obstacles for the normal teaching-learning process in classes), and (6) occasional participant students. This classification is based on the work of Roberts [33].

3) Teaching Strategy Questionnaire: Teachers were asked to provide the following information about their activities performed in their classes.

1) Learning activity type: The set of learning activity types was determined considering different areas such as nursing, sport lessons, electronics and automation engineering, computer science engineering, and psychology. In particular, the teachers should select among the following different types of activities:

- Hardware assembly: Each team of students assembles some hardware components collaboratively to conform a certain device.

- Software development: The students collaborate in teams, and each student develops some software component. For this purpose, students need to collaboratively establish certain interfaces, and strictly implement these. The students also need to coordinate so that different software components fit and properly work together. Students are advised to write readable programming code with the proper comments. 


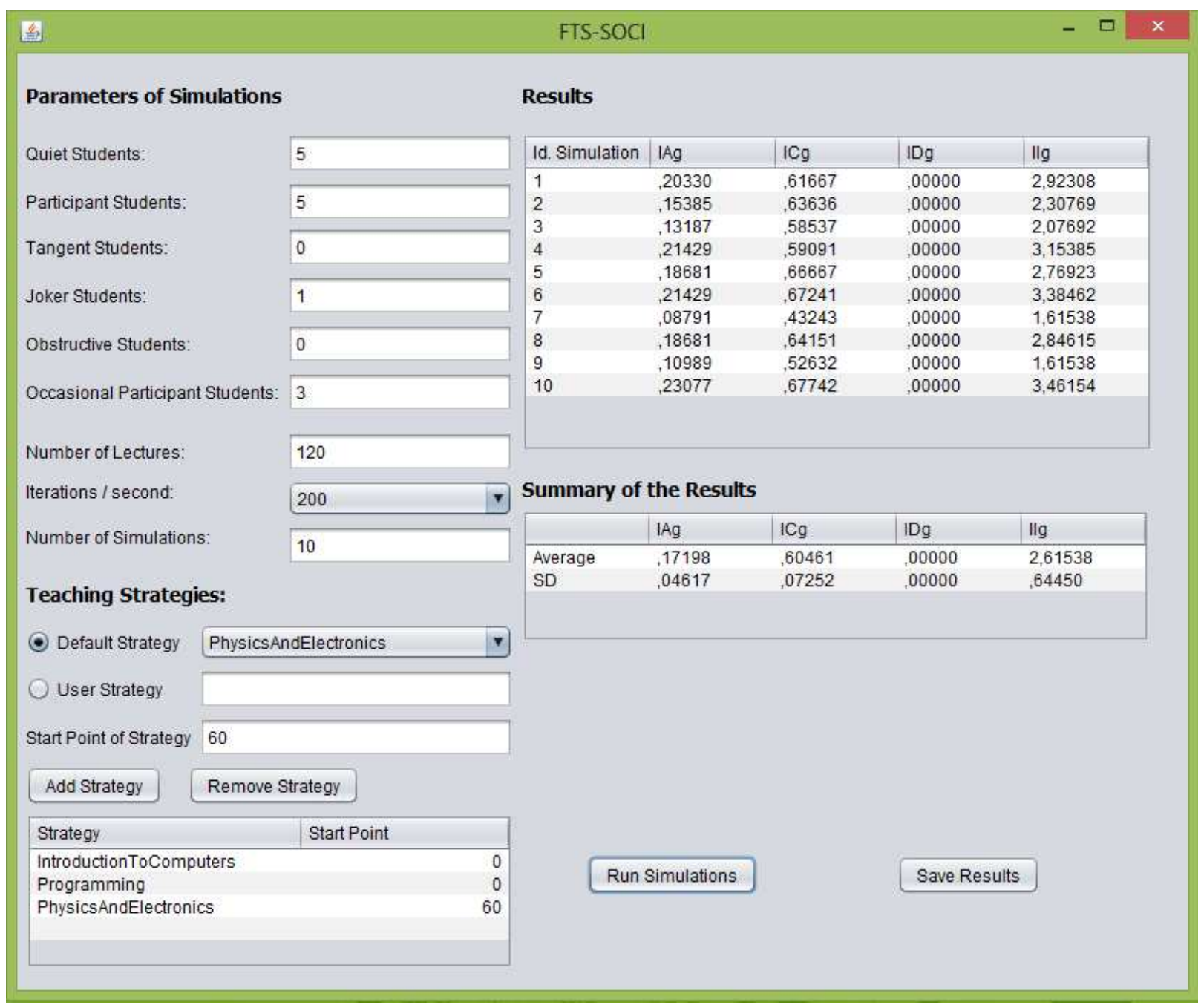

Fig. 2. User interface of the simulator of sociograms FTS-SOCI 2, which receives input from teaching strategies

- Document writing: Each team must write a coherent report in a collaborative way. To achieve this goal, firstly students need to agree on the structure and the way of presenting the ideas in the whole report. If the activity is designed to have a low level of interaction, normally each student writes a piece of the report, and then all the pieces are combined together in one document. One or several students revise the whole document for ensuring its coherency. If the activity is designed for a high level of interaction, commonly the group of students work most of the time together face-to-face, and they write the report in their meetings while discussing the most relevant aspects.

- Problem solving with public presentation: The teacher determines certain problem exercises and the students solve them either in groups or individually. Some students of some groups present their solutions to their classmates with the supervision of the teacher. Classmates can say comments or suggestions in the presentation.

- Problem solving with tutoring presentation: It is the same activity type as the previous one, but the presentation is performed only to the teacher in tutoring hours.

- Problem solving with no presentation: It is the same activity type as the two previous ones, but there is no presentation. Normally they submit their solution through the learning management system.

- Discussion: All the students of the class are invited to debate some topic selected by the teacher. Normally, the teacher highlights some relevant points or certain leading questions, so that the discussion activity is useful to think critically about the relevant aspects of the course. In this kind of activity, all the students interact with each other.

- Role-play: A group of students pretend to be certain characters in a particular situation indicated by the teacher. All the other students observe their performance and discuss about the situation and different actions.

2) The position in the timeline of the strategy: This information refers to the chronological-order position of the lecture in which the activity begins. 


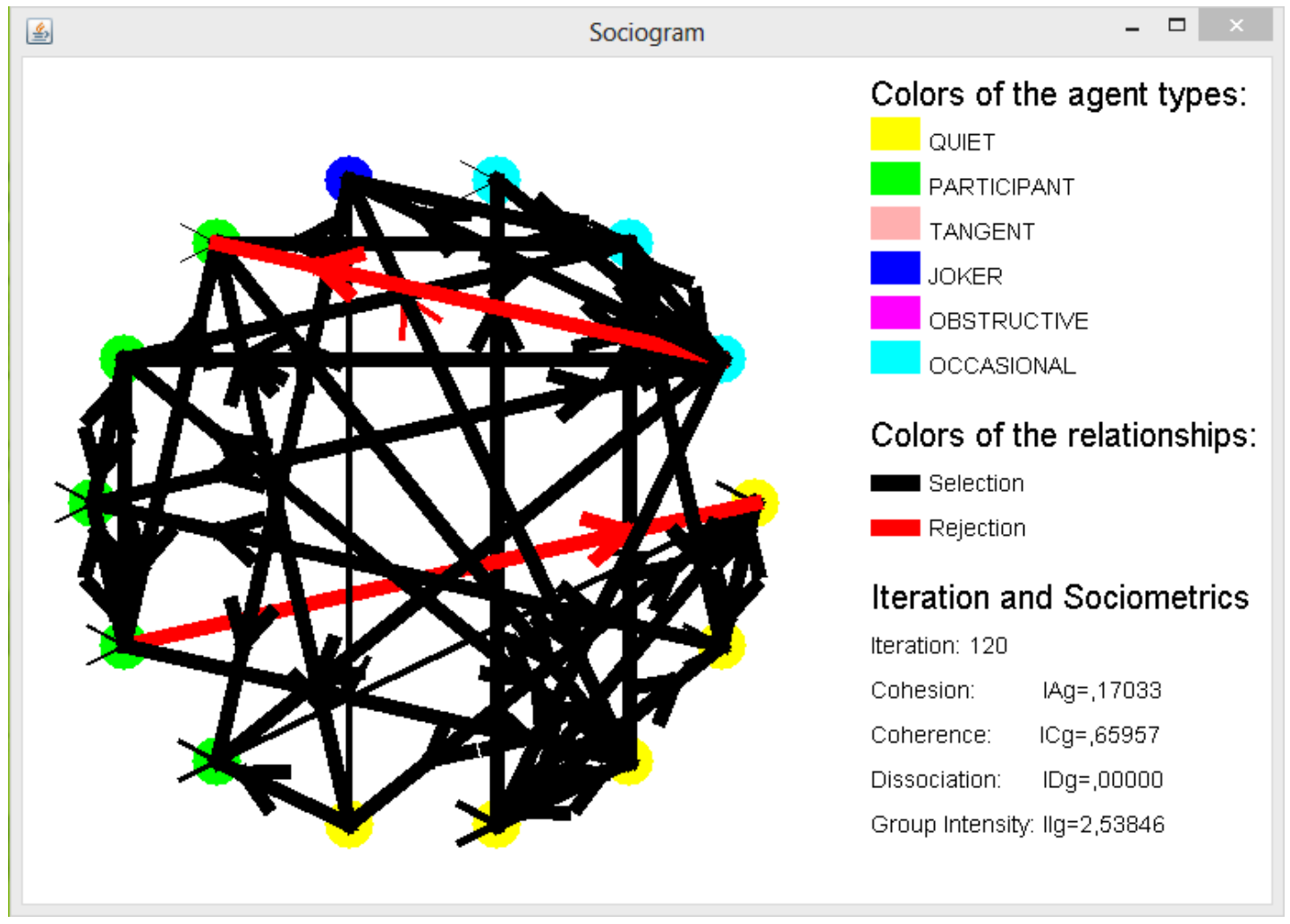

Fig. 3. Frame for showing the evolution of the sociogram in FTS-SOCI 2

3) The activity duration: This duration determines the number of hours that each student should work.

4) The size of teams when applicable: The number of students for each team.

5) The level of interaction: Teachers should decide if the learning activity involves high or low interaction among students. They should select "high" when they consider that the collaborative working time is estimated to be greater than the individual working time. If it is the other way around, they should select "low".

\section{Procedure}

The goal of the current work was introduced to the group of teachers from different disciplines in higher education. They were informed about the way of defining teaching strategies in FTS-SOCI 2, specially indicating the available kinds of learning activities and the necessary features for simulating these. In addition, we gave the survey and the instructions for the extraction of the sociograms with the behavioral types to the teachers.

Each of the teachers was asked to perform the experiment in one of their courses. The authors coordinated the experiments so that there were at least two courses that were taught simultaneously, two courses that were taught sequentially in the same academic course, and courses from different disciplines. Each of the teachers applied a particular teaching strategy of their choice in one course. Each teacher replied the questionnaire about teaching strategies (see section III-B3). At the end of the course, they asked the corresponding students to reply the surveys respectively about sociometric and classroom behavior, previously introduced in sections III-B1 and III-B2.

In order to reduce the possible negative impact of asking about personal social relations, the students were given the possibility to answer in an anonymous way, so that the person that transcribed the sociograms did not know the students' names. All the students' names were arranged in a list with identifiers. This list was available for students while filling the survey. Each student was asked to indicate their identifier and the ones of the students that they selected or rejected. After filling the survey, a person different from the teacher transcribed the answers without knowing the list of students. In this manner, nobody got informed about the personal social information of any student in particular. This procedure was explained to the students so that they could feel comfortable enough for replying the survey honestly.

In order to define the teaching strategies with FTS-SOCI 2, among other aspects in the teaching strategy questionnaire, the teachers selected which kind of collaborative exercises they had performed from the available list for each activity. The teachers were instructed to select the most similar exercise category, if some activity did not exactly fall under any of the available categories. Each strategy was defined with FTSSOCI 2 following the specific technical instructions previously introduced in II-A.

The sociometrics of these sociograms were calculated by representing these in the input format of one of the existing tools about sociograms called CLUS-SOCI [10], and this tool indicated the measurement values. FTS-SOCI 2 was evaluated by simulating nine different scenarios, including some with 
either consecutive or simultaneous teaching strategies. In order to avoid overfitting, first FTS-SOCI 2 was calibrated with three scenarios, and then it was validated with the other six different scenarios.

\section{RESUlts}

Table I shows the different scenarios simulated by FTSSOCI 2, and summarizes the strategies that the teachers had applied in the corresponding courses. In the scenarios with several courses, the table also indicates whether these were taught simultaneously in the same semester or consecutively in two different semesters. Notice that scenario 2 presents a oneyear experience composed of two courses in two consecutive semesters. In this case, the real outcomes were obtained from the responses of the survey collected at the end of the last course. In the case of simultaneous courses (i.e. scenario 3), the real outcomes were extracted from the survey at the end of one of the courses. All the courses included 60 lectures. The first three scenarios were used to calibrate the simulations performed by FTS-SOCI 2. All of these three scenarios involved courses in engineering grades. On the contrary, the last six scenarios were employed to validate the performance of FTS-SOCI 2 under scenarios not used in the calibration.

Table II determines the number of students of the group of each scenario (i.e. class size), and the number of students of each behavioral type in each scenario according to the selfreported information of the students.

Table III shows (a) the real sociometric values obtained from the data recollected by questionnaires, and (b) the values simulated by FTS-SOCI 2 in both calibration and validation. In particular, this tool performed 100 simulations, and calculated the simulated values by averaging the values obtained in the different simulations. The sociometric IIg was transformed into T-IIg for representing a proportion as in the other indices, by means of a division by the number of individuals.

Table IV provides the difference of proportions between the real and estimated values, as well as the two-tailed $\mathrm{p}$ value obtained with a binomial test. For the calibrated scenarios, all differences of proportions were close to 0.01 , except for some scenarios in the $\mathrm{ICg}$ index, in which the differences were higher. The same thing happened in the validated scenarios. Differences of proportions in calibrated scenarios were not significant, whereas only two differences in the validated scenario were significant: the ICg index for the Research Methodology course, and the IDg index for the Tourism course.

Table $\mathrm{V}$ presents the differences of the arcsine transformations of proportions (known as Cohen's h), a more appropriate effect size than the raw difference of proportions [34]. Following guidelines suggested by Cohen [34], 0.2, 0.5 and 0.8 were considered small, medium and large sizes respectively. The results showed that all the effect sizes were small, except the one for ICg index in Research Methodology course, which was a medium effect size.

With the purpose of knowing the predictive capability of FTS-SOCI 2 using a different statistic and the degree of the reliability of their simulations, we calculated the uncertainty coefficient (lambda) and Cohen's kappa, with their corresponding significance tests, by means of SPSS Statistics for Windows, Version 20.0 [35]. Table VI shows the results of these analyses. Uncertainty coefficient is a measure of association in terms of the proportional reduction in error when values of one variable are used to predict values of other variable. A value of zero means the predictor does not reduce any error, whereas a value of one indicates a complete reduction of error. Cohen's kappa measures the agreement of the evaluations of two raters for the same object. Kappa value ranges from zero (null agreement) to one (perfect agreement).

As a result of the low frequencies in some cells of the $2 \times 2$ table used to compute these measures, IDg index could not be calculated. Results revealed that for the calibrated scenarios all uncertainty coefficients were higher than 0.70. Respective to validated scenarios, uncertainty coefficients were higher than 0.76 for IAg and T-IIg indices, but lower for ICg index (specifically ranging from 0.37 to 0.60 ). In any case, all the uncertainty coefficients were significant, informing of a statistical proportional reduction in error. In a similar way, for the calibrated scenarios kappa values were higher than 0.80 in all indices, informing of an adequate reliability. Besides, whereas kappa values for validated scenarios were excellent for IAg and T-IIg indices, since they were higher than 0.90 respectively in five out of six scenarios in each of these sociometrics, kappa values for ICg index were not very good because they fell down below 0.80 value (specifically, ranging from 0.60 to 0.75 ). Although kappa values varied among indices, all of them were significant.

\section{Discussion}

The goal of the present work was to statistically test the predictive capacity of FTS-SOCI 2 to estimate social relationships (specifically, group sociometric indices) in higher education. Although similar ABSs were used by previous works [30], [31], in the present work we have introduced some improvements: (1) we have increased the number of learning activities to define teaching strategies, (2) we have modeled the influence of several teaching strategies over one group of students both simultaneous and consecutively, (3) we have distinguished computationally between selection and rejections in the definition of the internal constants about the coherence of reciprocal relations, to improve accuracy of predictions, (4) we have made the introduction of the sequence of strategies more user-friendly, by indicating its duration in weeks or months, instead of through its temporal position, and (5) we have evaluated the predictive capacity of FTS-SOCI 2 in courses that were not used during the calibration phase. Whereas some of this courses belonged to engineering degree, as those courses used in calibration phase, others belonged to different types of disciplines, which were psychology, business, tourism, and renewal energies.

Taking both calibrated and validated data together, results showed that the majority of the differences of proportions (75\% of the data) between the real and estimated values were close to 0.01 . Values higher than this were exclusively found in ICg index, except one exception in T-IIg. Based on Cohen's h effect sizes, all differences can be interpreted as 
TABLE I

SiMULATED SCENARIOS

\begin{tabular}{|c|c|c|}
\hline Id. & Scenario & Summary of Teaching Strategy \\
\hline 1 & "Introduction to Computers" & $\begin{array}{l}\text { The teacher asked students to perform five teamwork activities in pairs and one teamwork activity in groups of four members. All } \\
\text { these teamwork activities were about collaborative assembly of hardware components, and required a high level of interaction. In } \\
\text { addition, the students were asked to solve certain problems and perform public presentations, and in this activity all the students } \\
\text { worked collaboratively together with a low level of interaction. Finally, the students worked in pairs for solving certain problems } \\
\text { and presenting the solutions in tutoring time. }\end{array}$ \\
\hline 2 & $\begin{array}{l}\text { One-year experience with subjects } \\
\text { Introduction to Computers and } \\
\text { "Physics and Electronics" in con- } \\
\text { secutive semester }\end{array}$ & $\begin{array}{l}\text { The teaching strategy of Physics and Electronics only had three collaborative activities for respectively hardware assembly, software } \\
\text { development and problem solving with tutoring presentation. In these activities, the students worked in pairs with a high level of } \\
\text { interaction. The teaching strategy of Introduction to Computers was introduced in the previous scenario. }\end{array}$ \\
\hline 3 & $\begin{array}{l}\text { Both "Programming 1" } \\
\text { Physics and Electronics in the } \\
\text { same semester }\end{array}$ & $\begin{array}{l}\text { In Programming 1, the main learning experience was software development in pairs with three long activities of } 15 \text { hours each } \\
\text { one. Students also individually performed certain exercises with public presentations in which students interacted. Finally, the } \\
\text { teacher asked students to discuss about a certain topic. The teaching strategy of Physics and Electronics was introduced in the } \\
\text { previous scenario. }\end{array}$ \\
\hline 4 & "Electrotechnics" & $\begin{array}{l}\text { This teacher asked five practices of hardware assemblies in pairs and three works about solving problems and presenting the } \\
\text { solutions in tutoring hours. The practices required different levels of interactions depending on their goals, whereas in the works } \\
\text { all the students actively worked together. }\end{array}$ \\
\hline 5 & "Research Methodology" & $\begin{array}{l}\text { In this course, there were two discussion activities at the beginning, and then the students performed short exercises every week } \\
\text { without presentations. }\end{array}$ \\
\hline 6 & "Management Techniques" & In this course, the collaboration of students were teamwork in groups in some sessions. \\
\hline 7 & "Tourism" & The teacher asked students to do short teamwork activities every session in pairs, and they also had a few discussions. \\
\hline 8 & "Renewable Energies" & The students performed teamwork activities about document writing in pairs in some sessions. \\
\hline 9 & "Software Engineering" & The teacher combined activities of software development and document writing. \\
\hline
\end{tabular}

TABLE II

Class Sizes AND STUdent Types

\begin{tabular}{llllllll}
\hline Case & Class size & Quiet & Participant & Tangent & Joker & Obstructive & Occasional Participant \\
\hline 1 & 47 & 8 & 13 & 0 & 3 & 0 & 23 \\
2 & 30 & 4 & 11 & 0 & 3 & 0 & 12 \\
3 & 12 & 3 & 3 & 0 & 1 & 0 & 5 \\
4 & 11 & 0 & 3 & 0 & 3 & 0 & 17 \\
5 & 38 & 12 & 7 & 0 & 2 & 0 & 29 \\
6 & 42 & 1 & 12 & 0 & 0 & 0 & 19 \\
7 & 61 & 4 & 33 & 0 & 4 & 1 & 5 \\
8 & 11 & 1 & 5 & 0 & 1 & 0 & 5 \\
9 & 29 & 8 & 13 & 0 & 2 & 1 & \\
\hline
\end{tabular}

TABLE III

REAL AND Estimated VALues

\begin{tabular}{|c|c|c|c|c|c|c|c|c|c|c|c|}
\hline \multirow[b]{2}{*}{ Phase } & \multirow[b]{2}{*}{ Case } & \multicolumn{5}{|c|}{ Real value } & \multicolumn{5}{|c|}{ Simulated Value } \\
\hline & & IAg & $\mathrm{ICg}$ & IDg & IIg & T-IIg & IAg & $\mathrm{ICg}$ & IDg & IIg & T-IIg \\
\hline \multirow{3}{*}{ Calibration } & 1 & 0.03330 & 0.43636 & 0.00000 & 1.97826 & 0.04209 & 0.03289 & 0.44131 & 0.00016 & 2.00087 & 0.04257 \\
\hline & 2 & 0.04598 & 0.41667 & 0.00230 & 2.06897 & 0.06897 & 0.04532 & 0.47817 & 0.00030 & 1.73345 & 0.05778 \\
\hline & 3 & 0.13636 & 0.54545 & 0.00000 & 2.09091 & 0.17424 & 0.14924 & 0.58013 & 0.00000 & 1.94545 & 0.16212 \\
\hline \multirow{6}{*}{ Validation } & 4 & 0.12727 & 0.63636 & 0.00000 & 1.70000 & 0.15455 & 0.13682 & 0.50944 & 0.00036 & 1.65200 & 0.15018 \\
\hline & 5 & 0.08321 & 0.63587 & 0.00000 & 3.54054 & 0.09317 & 0.07503 & 0.80262 & 0.00071 & 3.29216 & 0.08664 \\
\hline & 6 & 0.00946 & 0.34307 & 0.00161 & 1.55714 & 0.03707 & 0.01103 & 0.29008 & 0.00168 & 1.66022 & 0.03953 \\
\hline & 7 & 0.01104 & 0.31481 & 0.00227 & 3.15385 & 0.05170 & 0.01176 & 0.41233 & 0.00000 & 2.97445 & 0.04876 \\
\hline & 8 & 0.05455 & 0.60000 & 0.01818 & 1.20000 & 0.10909 & 0.04532 & 0.57008 & 0.00998 & 1.44322 & 0.13120 \\
\hline & 9 & 0.01011 & 0.27820 & 0.00328 & 1.56667 & 0.05402 & 0.01202 & 0.40310 & 0.00274 & 1.74430 & 0.06015 \\
\hline
\end{tabular}

TABLE IV

Differences and Comparison with the BinOMial Test. *** Significant With a 0.001 Level

\begin{tabular}{|c|c|c|c|c|c|c|c|c|c|}
\hline \multirow[b]{2}{*}{ Phase } & \multirow[b]{2}{*}{ Case } & \multicolumn{4}{|c|}{ Differences } & \multicolumn{4}{|c|}{ Binomial test (two-tailed p-value) } \\
\hline & & IAg & $\mathrm{ICg}$ & IDg & T-IIg & $\mathrm{IAg}$ & $\mathrm{ICg}$ & $\mathrm{IDg}$ & T-IIg \\
\hline \multirow{3}{*}{ Calibration } & 1 & -0.00041 & 0.00495 & 0.00016 & -0.00048 & 0.968 & 0.897 & 0.707 & 0.860 \\
\hline & 2 & -0.00066 & 0.06150 & -0.00200 & 0.01118 & 0.955 & 0.291 & 0.770 & 0.199 \\
\hline & 3 & 0.01288 & 0.03468 & 0.00000 & 0.01212 & 0.684 & 0.606 & 1.000 & 0.748 \\
\hline \multirow{6}{*}{ Validation } & 4 & 0.00955 & -0.12692 & 0.00036 & 0.00436 & 0.647 & 0.565 & 0.961 & 0.919 \\
\hline & 5 & -0.00818 & 0.16675 & 0.00071 & 0.00654 & 0.266 & $0.000^{* * * *}$ & 0.368 & 0.385 \\
\hline & 6 & 0.00157 & -0.05299 & 0.00007 & -0.00245 & 0.563 & 0.852 & 0.952 & 0.544 \\
\hline & 7 & 0.00072 & 0.09752 & -0.00227 & 0.00294 & 0.611 & 0.109 & $0.000 * * *$ & 0.425 \\
\hline & 8 & -0.00923 & -0.02992 & -0.00820 & -0.02211 & 0.555 & 0.259 & 0.807 & 0.434 \\
\hline & 9 & 0.00191 & 0.12490 & -0.00054 & -0.00613 & 0.618 & 0.149 & 0.995 & 0.465 \\
\hline
\end{tabular}

small effect sizes, except that for the ICg index in one of the validated scenarios (specifically, Research Methodology course), in which the effect size was medium and significant. All these results advocate that FTS-SOCI 2 predicts IAg, IDg and T-IIg indices well, being the prediction worse in ICg index, especially in one of the validated scenarios.

With regards to the quantification of the predictive capability in terms of proportional reduction in error, uncertainty coefficients were higher than 0.70 and significant for both calibrated and validated scenarios. This cannot be applied to ICg index for the validated scenarios, whose values were lower, but 
TABLE V

DifFERENCES OF ARCSINE TRANSFORMATIONS OF PROPORTIONS (COHEN'S H)

\begin{tabular}{llllll}
\hline Phase & Case & IAg & ICg & IDg & T-IIg \\
\hline \multirow{3}{*}{ Calibration } & 1 & 0.002 & 0.010 & 0.025 & 0.002 \\
& 2 & 0.003 & 0.124 & 0.061 & 0.046 \\
\hline \multirow{2}{*}{ Validation } & 3 & 0.037 & 0.070 & 0.000 & 0.032 \\
& 4 & 0.028 & 0.257 & 0.038 & 0.012 \\
& 5 & 0.030 & 0.375 & 0.053 & 0.023 \\
& 7 & 0.016 & 0.114 & 0.002 & 0.013 \\
& 8 & 0.007 & 0.203 & 0.095 & 0.013 \\
& 9 & 0.042 & 0.061 & 0.070 & 0.068 \\
& 0.018 & 0.265 & 0.010 & 0.026 \\
\hline
\end{tabular}

TABLE VI

UNCERTAINTY COEFFICIENT AND COHEN'S KAPPA. *** SigNIFICANT WITH A 0.001 LEVEL

\begin{tabular}{|c|c|c|c|c|c|c|c|c|c|}
\hline \multirow[b]{2}{*}{ Phase } & \multirow[b]{2}{*}{ Case } & \multicolumn{4}{|c|}{ Uncertainty coefficient } & \multicolumn{4}{|l|}{ Kappa } \\
\hline & & IAg & $\mathrm{ICg}$ & IDg & T-IIg & IAg & $\mathrm{ICg}$ & IDg & T-IIg \\
\hline \multirow{3}{*}{ Calibration } & 1 & $0.973 * * *$ & $0.959 * * *$ & - & $0.985 * * *$ & $0.993 * * *$ & $0.990^{* * *}$ & - & $0.994 * * *$ \\
\hline & 2 & $0.952 * * *$ & $0.732 * * *$ & - & $0.753^{* * *}$ & $0.987 * * *$ & $0.877 * * *$ & - & $0.903^{* * *} *$ \\
\hline & 3 & $0.876 * * *$ & $0.797 * * *$ & - & $0.836^{* * *}$ & $0.939 * * *$ & $0.923 * * *$ & - & $0.945^{* * *}$ \\
\hline \multirow{6}{*}{ Validation } & 4 & $0.912 * * *$ & $0.571 * * *$ & - & $1.000^{* * *}$ & $0.960^{* * *}$ & $0.744 * * *$ & - & $1.000^{* * *}$ \\
\hline & 5 & $0.831 * * *$ & $0.375 * * *$ & - & $0.877 * * *$ & $0.941 * * *$ & $0.602 * * *$ & - & $0.961^{* * *} *$ \\
\hline & 6 & $0.808 * * *$ & $0.394 * * *$ & - & $0.812^{* * *}$ & $0.931 * * *$ & $0.634 * * *$ & - & $0.902 * * *$ \\
\hline & 7 & $0.872 * * *$ & $0.596 * * *$ & - & $0.932 * * *$ & $0.942 * * *$ & $0.750 * * *$ & - & $0.954 * * *$ \\
\hline & 8 & $0.822 * * *$ & $0.398 * * *$ & - & $0.768 * * *$ & $0.833^{* * *}$ & $0.715 * * *$ & - & $0.851 * * *$ \\
\hline & 9 & $0.742 * * *$ & $0.421 * * *$ & - & $0.794 * * *$ & $0.914 * * *$ & $0.617 * * *$ & - & 0.930 *** \\
\hline All & & $0.884 * * *$ & $0.671 * * *$ & - & $0.862 * * *$ & $0.925^{* * *}$ & $0.866 * * *$ & - & 0.958 *** \\
\hline
\end{tabular}

significant. Another exception was found in IDg index, whose values could not be calculated as a consequence of very low frequencies. Analogous results were found in Cohen's kappa values. Concretely, Cohen's kappa values were higher than 0.80 and significant in all indices, showing adequate reliabilities, except in the ICg index for the validated scenarios, whose values were lower and also significant, and in IDg index due to Cohen's kappa could not be calculated.

FTS-SOCI 2 was designed to predict sociometric indices, which were based on the model proposed by Dunkin and Biddle [26]. Consequently, our results have implications for this model. According to this model, both student-related variables and teacher-related variables determine the interactive behaviors in the classroom. Our results confirmed this prediction in all the analyzed sociometrics. There was only one exception out of nine scenarios for ICg and another exception out of nine scenarios for IDg, according to the binomial test results. Thus, our findings are in concordance with the background educational theory of Dunkin and Biddle.

A further point for consideration is that results obtained with calibrated data were parallel to those results observed with validated data, which provides generality to the predictability of FTS-SOCI 2.

Although students were explicitly asked to choose only one student type, some students found trouble in classifying themselves in only one category. More concretely, four students chose two student types for determining their behavioral profile. In these combined answers, the most frequent profiles were joker and occasional participant, which were combined together or with other profiles. This fact is shared with other fields that consider psychological features. People can behave, feel, and perceive in combinations of different ways. For example, in the field of emotions, Ekman [36] argues that it is useful to represent emotions as combinations of some basic ones.
None of teachers indicated that they missed any exercise category when they had participated in this experimentation. However, designing teaching strategies can involve many different kinds of exercises. Although in this experimentation the six teachers did not find trouble in classifying the collaborative activities in the categories provided in the simulator, we cannot guarantee that other teachers will certainly find the list of exercise categories appropriate. Thus, this simulator may be improved in this aspect when more teachers use the proposed simulator and provide feedback.

Teaching strategies are dependent of disciplines and their nature, and consequently the nature of collaborative activities also varies regarding the discipline. In order to support the definition and simulation of teaching strategies, we have defined some exercise categories that are common to most disciplines, like discussions in classrooms or collaborative document writing. In addition, different levels of interaction and durations can be determined, so that these categories can be properly used for activities in different disciplines. In order to define some particularities of a few disciples, such as collaborative software development or hardware assembly, we have also defined specific collaborative activities. In the experiments, the simulator was calibrated in the disciplines of computer science and electronics, and then it was validated in other subjects belonging to the disciplines of psychology, business, tourism, and renewal energies. Since the validation results were appropriate in disciplines different from the ones used in the calibration, these results support the crossdiscipline validity of this tool despite the different nature of collaboration activities in different disciplines. The similarity between real and simulated outcomes in the different disciplines can be observed in the results of the binomial test between real and simulated values. In particular, there were no significant differences between real and simulated outcomes in the cohesion (IAg) and intensity (T-IIg) sociometrics in all 
the nine scenarios belonging to the different disciplines. In the case of coherence (ICg) and dissociation (IDg) sociometrics, there were no significant differences in eight out of the nine scenarios. The exception scenario with significant differences in cohesion was different from the exception scenario for dissociation. In particular, the scenarios of all the disciplines obtained similar results in the four sociometrics, except the scenario of the psychology discipline that performed properly in three out of the four sociometrics and the scenario in tourism discipline that performed properly in other three out of four sociometrics.

It is worth mentioning that five scenarios of this study were performed during the academic year 2015-2016, and other four scenarios were performed in the academic year 2018-2019. The purpose of the experiments of the latter academic year was to determine whether the results obtained in the former academic year could be reproduced, considering both a subject from the same discipline (in this case computer science), and subjects from different disciplines (business, tourism, and renewable energy), in a different institution (Cadi Ayyad University instead of University of Zaragoza) in a different country (Morocco instead of Spain). Based on the binomial test, in the latter experimentation there were no significant differences between real and simulated outcomes in all the scenarios and all the sociometrics except one sociometric in one scenario, and these results were similar to the former experimentation (all sociometrics in all scenarios without significant differences except one sociometric in one scenario). Therefore, the latter experiments reproduced the findings of the former experiments, three academic years later.

In general, all this experimentation was performed for helping teachers in deciding among different teaching strategies for a particular group of students. The goal was that teachers could test several teaching strategies (including the organization of activities) with the simulator for a particular group of students, in order to determine which strategy may imply a higher cohesion among students, and consequently probably a higher academic performance. For example, some teachers observed that the organization of activities played a key role, having a greater impact the collaborative activities that were scheduled earlier in the teaching plan. The results of the simulator can also affect the teaching organization concerning the creation of student groups for the classes of a given subject. The simulator could test a particular teaching strategy in different configurations of student groups, for determining which one may be the most appropriate for achieving an adequate cohesion in average.

Although this study was performed in universities of two different countries with different cultures (Spain and Morocco), the number of countries and cultures is not enough to prove that the current ABS approach is useful in all the countries and cultures, since this number is not representative. In addition, to ensure a high impact of this approach, the study would have needed to also focus on the users' experience (i.e. teachers' experience). In this way, we would have evaluated if the tool is perceived as easy-to-use, intuitive and useful enough to make this tool widespread if properly disseminated.

\section{CONClusions AND Lines OF FUture WORK}

The results provide evidence for replying the research questions of this work. First, the sociometric status (represented by a sociogram) is an aspect that can be predicted from teaching strategies, and this work shows it with a novel study. Second, the proposed ABS has proven to be useful for predicting group indices of social relations in a general context of higher education. The findings of the current approach advocates to be general, since the presented experiments cover six different disciplines, which are computer science engineering, electronics and automation engineering, psychology, business and management, tourism, and renewal energies.

An agent-based social simulation tool has been developed for simulating the repercussion of teaching strategies of higher education on the sociograms of particular groups of students. The students can be categorized into six behavioral types. The tool provides both a graphical visualization of the simulated sociogram and the estimation of several group sociometrics. The teaching strategies can be defined considering up to eight different kinds of common activities. Our results have shown that based on differences of proportions, effect sizes and uncertainty coefficients, the predictive capacity of FTSSOCI 2 to estimate sociometric indices were good in IAg, IDg and T-IIg indices, being lower in the ICg index, although still adequate. This reduction of the predictive capacity was more profound in the validated scenarios, suggesting certain degree of overfitting in the ICg index for the calibrated scenarios. Likewise, based on Cohen's kappa values, FTSSOCI 2 simulations provide estimations that are reliable for IAg and T-IIg indices. And, although Cohen's kappa values were also adequate in $\mathrm{ICg}$ index for the calibrated scenario, the reduction in reliability found in the IDg index for the validated scenarios seems to be the product of overfitting. Finally, the impossibility of calculation of uncertainty coefficients and Cohen's kappa values is not only a technical problem. It is also the reflection of the difficulty of reducing error under conditions where the occurrence of an event is very low. This does not happen when predictive capacity is assessed through the difference between real and predicted values (i.e., through difference of proportions and Cohen's h).

There are some limitations of this study that need to be considered. To determine student classroom behavior, students were classified in different types of classroom behaviors. Unlike in a previous study [30] where student behavior was evaluated by the teacher, in the present study students selfassessed their own behavior. This is problematic since students may classify themselves to a category of behavior that does not correspond to them for an effect of social desirability. Future research could overcome this limitation obtaining this information using teacher assessment and/or peers' assessment.

Another limitation of the current work is that the usability of the tool has not been tested. The usability will be assessed after we introduce certain improvements. We are currently designing the development of an easy-to-use interface where users can configure their teaching strategies by dragging and dropping certain visual elements representing learning activities. In addition, the tool is planned to be converted 
into both a mobile application and an online tool, following TABSAOND (a Technique for developing Agent-Based Simulation Apps and Online tools with Nondeterministic Decisions) [37]. All these tools will incorporate the possibility of sending certain information and feedback to the authors, so that more experiences can be analyzed from different universities and countries.

\section{APPENDIX}

\section{DETAILED DESCRIPTIONS OF THE USED SOCIOMETRICS}

The equations of the used sociometrics were already introduced by their authors [32]. This section only introduces the basic principles and describes their meaning in the context of the current work.

Initially, [32] defined some individual metrics concerning the selections and rejections received by each user, for later defining the group sociometrics. They distinguished between reciprocal relations and the others, since the former ones are usually more meaningful for detecting some properties of the group.

The cohesion (IAg) focuses on the ratio between the existing reciprocal relations and the possible relations (i.e. all the paired combinations between each other). The cohesion is useful for comprehending whether students normally rely on each other within a group. In other words, it represents the ratio of reciprocal acceptance among students.

The dissociation (IDg) represents the opposite to the previous metrics, since it is centered on the ratio between the reciprocal rejections and the number of all possible paired combinations. This metric can detect the average ratio of reciprocal rejections, and can detect problems in a students' group.

The coherence $(\mathrm{ICg})$ refers to the ratio between reciprocal selections and the selections received by other students. In other words, it represents the ratio of reciprocity in the selections of students. This metric can be useful for detecting whether students tend to have reciprocal relations.

The density (IIg) measures the percentage of relations (either reciprocal or not) among the number of possible paired combinations. Sometimes, it can be useful to measure how much the students are related regardless it is positively or negatively. Normally, a high density means that students know well each other.

\section{ACKNOWLEDGMENT}

The current work has been mainly supported by the project "Diseño curricular de asignaturas con actividades colaborativas asistido con un simulador basado en agentes" with reference PIIDUZ_15_193 and funded by the University of Zaragoza. This work acknowledges the research project "Construcción de un framework para agilizar el desarrollo de aplicaciones móviles en el ámbito de la salud" funded by University of Zaragoza and Foundation Ibercaja with grant reference JIUZ-2017-TEC-03. It has also been supported by "Organismo Autónomo Programas Educativos Europeos" with reference 2013-1-CZ1-GRU06-14277. This work has also been supported by the program "Estancias de movilidad en el extranjero José Castillejo para jóvenes doctores" funded by the Spanish Ministry of Education, Culture and Sport with reference CAS17/00005. We also acknowledge support from "Universidad de Zaragoza", "Fundación Bancaria Ibercaja" and "Fundación CAI" in the "Programa Ibercaja-CAI de Estancias de Investigación" with references IT24/16 and IT1/18. Furthermore, we acknowledge the "Dpto. de Innovación, Investigación y Universidad del Gobierno de Aragón" for their support through the program "FEDER Aragón 2014-2020 Construyendo Europa desde Aragón" granted to EduQTech research group (Ref: T49_17R), which funded the research stay of the first author in Massachusetts General Hospital and Harvard University in the last stages of this work. We also acknowledge the support of the projects "Collaborative Ambient Assisted living Design" (TIN2014-57028-R), "Diseño colaborativo para la promoción del bienestar en ciudades inteligentes inclusivas" (TIN2017-88327-R), and "Red Temática de Investigación en Ciudades Inteligentes" (TIN2016-81766REDT) funded by the Spanish council of Science, Innovation and Universities from the Spanish Government..

\section{REFERENCES}

[1] M. Kim and S. Kim, "Debriefing practices in simulation-based nursing education in south korea," Clin. Simul. Nursing, vol. 13, no. 5, pp. 201209, May 2017.

[2] E. A. Swanson, A. C. Nicholson, T. A. Boese, E. Cram, A. M. Stineman, and K. Tew, "Comparison of selected teaching strategies incorporating simulation and student outcomes," Clin. Simul. Nursing, vol. 7, no. 3, pp. e81-e90, May 2011.

[3] L. G. Kothari, K. Shah, and P. Barach, "Simulation based medical education in graduate medical education training and assessment programs," Prog. Pediatric Cardiology, vol. 44, pp. 33-42, Mar. 2017.

[4] R. J. Lancaster, "Serious game simulation as a teaching strategy in pharmacology," Clin. Simul. Nursing, vol. 10, no. 3, pp. e129-e137, Mar. 2014.

[5] F. Borrajo, Y. Bueno, I. De Pablo, B. Santos, F. Fernández, J. García, and I. Sagredo, "SIMBA: A simulator for business education and research," Decis. Support Syst., vol. 48, no. 3, pp. 498-506, Feb. 2010.

[6] R. Pozo-Barajas, M. del Pópulo Pablo-Romero, and R. Caballero, "Evaluating a computer-based simulator program to teach the principles of macroeconomic equilibria," Comput. Edu., vol. 69, pp. 71-84, Nov. 2013.

[7] F. Ke, S. Lee, and X. Xu, "Teaching training in a mixed-reality integrated learning environment," Comput. Human Behav., vol. 62, pp. 212-220, Sep. 2016.

[8] S. P. Brubacher, M. Powell, H. Skouteris, and B. Guadagno, "The effects of e-simulation interview training on teachers' use of open-ended questions," Child Abuse Neglect, vol. 43, pp. 95-103, May 2015.

[9] J. L. Moreno, Sociometry, Experimental Method and the Science of Society. An Approach to a New Political Orientation. New York: Beacon House, Beacon, 1951

[10] I. García-Magariño, C. Medrano, A. S. Lombas, and A. Barrasa, "A hybrid approach with agent-based simulation and clustering for sociograms," Inf. Sci., vol. 345, pp. 81-95, Jun. 2016.

[11] S. Abar, G. K. Theodoropoulos, P. Lemarinier, and G. M. O'Hare, "Agent based modelling and simulation tools: A review of the stateof-art software," Comput. Sci. Rev., vol. 24, pp. 13-33, May. 2017.

[12] S. Ioan Sabin, S. Mihai, and P. Marcel, "The importance and utility of the sociometric survey method in physical education research," Procedia - Social Behav. Sci., vol. 117, pp. 185 - 192, Mar. 2014.

[13] J. Jostad, J. Sibthorp, and K. Paisley, "Understanding groups in outdoor adventure education through social network analysis," J. Outdoor Environ. Edu., vol. 17, no. 1, pp. 17-31, Oct. 2013.

[14] S. Petracovschi, P. Runcan, R. Neniu, and G. Clitan, "The social integration of children with weight issues using the curricular and extracurricular physical education activities," Procedia - Social Behav. Sci., vol. 46, pp. 2872 - 2876, Feb. 2012.

[15] C. Borch, A. Hyde, and A. H. Cillessen, "The role of attractiveness and aggression in high school popularity," Social Psychol. Edu., vol. 14, no. 1, pp. 23-39, Mar. 2011. 
[16] M. J. Zimmer-Gembeck, R. E. Pronk, B. Goodwin, S. Mastro, and N. R. Crick, "Connected and isolated victims of relational aggression: Associations with peer group status and differences between girls and boys," Sex Roles, vol. 68, no. 5-6, pp. 363-377, Mar. 2013.

[17] K. L. Bierman, Peer rejection: Developmental processes and intervention strategies. New York, NY: Guilford Press, 2004.

[18] A. F. Newcomb, W. M. Bukowski, and L. Pattee, "Children's peer relations: a meta-analytic review of popular, rejected, neglected, controversial, and average sociometric status." Psychol. Bull., vol. 113, no. 1, p. 99, Jan. 1993.

[19] C. Howe, Peer groups and children's development. Oxford, UK: WileyBlackwell, 2010.

[20] L. O. Gallardo and A. Barrasa, "Analysis of the changing relationship between peer acceptance and academic achievement in adolescents/análisis de la relación variable entre la aceptación entre iguales y el rendimiento académico de los adolescentes," Revista Psicología Social, vol. 31, no. 3 , pp. 589-608, Mar. 2016.

[21] L. O. Gallardo, A. Barrasa, and F. Guevara-Viejo, "Positive peer relationships and academic achievement across early and midadolescence," Social Behav. Personality: Int. J., vol. 44, no. 10, pp. 1637-1648, Nov. 2016.

[22] C. A. Haythornthwaite, "Learning relations and networks in web-based communities," Int. J. Web Based Communities, vol. 4, no. 2, p. 140-158, Nov. 2008.

[23] J. Zhao, "A sociogram analysis on group interaction in an online discussion forum," in Adv. Web Based Learn., F. Li, J. Zhao, T. K. Shih, R. Lau, Q. Li, and D. McLeod, Eds. Berlin, Heidelberg: Springer, 2008, pp. 377-389.

[24] L. P. Macfadyen and S. Dawson, "Mining LMS data to develop an "early warning system" for educators: A proof of concept," Comput. Edu., vol. 54, no. 2, pp. 588 - 599, Feb. 2010.

[25] S. Dawson, "Seeing' the learning community: An exploration of the development of a resource for monitoring online student networking," Brit. J. Edu. Technol., vol. 41, no. 5, pp. 736-752, 2010.

[26] M. J. Dunkin and B. J. Biddle, The study of teaching. New York: Holt, Rinehart \& Winston, 1974.

[27] A. J. Martin and M. Dowson, "Interpersonal relationships, motivation, engagement, and achievement: Yields for theory, current issues, and educational practice," Rev. Edu. Res., vol. 79, no. 1, pp. 327-365, Mar 2009.

[28] G. W. Ladd, "Having friends, keeping friends, making friends, and being liked by peers in the classroom: Predictors of children's early school adjustment?" Child Develop., vol. 61, no. 4, pp. 1081-1100, Aug. 1990

[29] J. G. Parker and S. R. Asher, "Peer relations and later personal adjustment: Are low-accepted children at risk?" Psychol. Bull., vol. 102, no. 3, p. 357, Dec. 1987.

[30] I. García-Magariño and I. Plaza, "FTS-SOCI: An agent-based framework for simulating teaching strategies with evolutions of sociograms," Simul. Model. Pract. Theory, vol. 57, pp. 161-178, Sep. 2015.

[31] I. García-Magariño, I. Plaza, R. Igual, and A. S. Lombas, "Designing teaching strategies with an agent-based simulator," in Model. Simul. Eng. Econ. Manage., ser. Lecture Notes Bus. Inf. Process. Springer, Jun. 2016, vol. 254, pp. 176-185.

[32] A. Barrasa and F. Gil, "A software application for the calculus and representation of sociometric indexes and values (In Spanish Un programa informático para el cálculo y la representación de índices y valores sociométricos)," Psicothema, vol. 16, no. 2, pp. 329-335, Jan. 2004.

[33] S. Roberts, "Using practitioner research to investigate the role of the teacher in encouraging student interaction within group work," Nurse Edu. Today, vol. 28, no. 1, pp. 85-92, Jan. 2008.

[34] J. Cohen, Statistical power analysis for the behavioral sciences (2nd ed.). Hillsdale, NJ: Lawrence Erlbaum Associates, 1988.

[35] IBM Corp., IBM SPSS statistics for Windows, version 20.0. New York: IBM Corp, 2011.

[36] P. Ekman, "Are there basic emotions?" Psychol. Rev., vol. 99, pp. 550553, Jul. 1992.

[37] I. García-Magariño, G. Palacios-Navarro, and R. Lacuesta, "TABSAOND: A technique for developing agent-based simulation apps and online tools with nondeterministic decisions," Simul. Model. Pract. Theory, vol. 77, pp. 84-107, Sep. 2017.

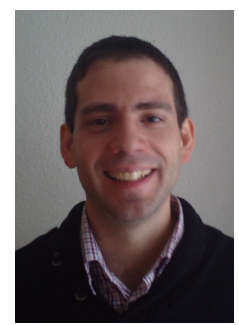

Iván García-Magariño is a lecturer and a contributor to the GRASIA research group at the Complutense University of Madrid. Prior to commencing this position in 2018, he was a $\mathrm{PhD}$ assistant professor in University of Zaragoza (2014-2018), and before he was a lecturer at the Madrid Open University (2010-2014). Iván was awarded his Ph.D. in Computer Science Engineering from the Complutense University of Madrid in 2009, and was a recipient of an FPI scholarship from 2006 to 2010. His main research interests include agent-based simulators, multi-agent systems, and agent-oriented software engineering. Among journals, book chapters, conferences and workshops, he has over 115 publications (over 48 in journals with ISI Thomson JCR). He actively collaborates with the EduQTech research group from the University of Zaragoza on $\mathrm{m}$-health projects, the HCI Lab from the University of Udine (Italy) on human-computer interaction projects, Institute of Technology Blanchardstown (Ireland) on datamining projects, and the Multicultural Alzheimer Prevention Program (MAPP) of Massachusetts General Hospital and Harvard University (US) on m-health projects. He is editor in several special issues with impact factor.

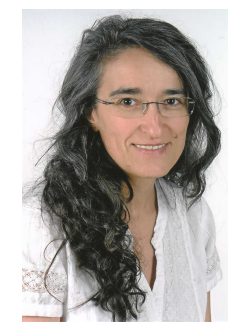

Inmaculada Plaza (SM'06) obtained her degree in Physics, the DEA in Manufacturing Engineering and $\mathrm{a} \mathrm{PhD}$ in the department of Electronics and Communication. Accredited as full professor in "Engineering and Architecture" (2016). She founded the technical group EduQTech and the chair in "Innovation and Technical Quality". She is the leader of 11 collaboration agreements and the main researcher of 27 projects (regional, national and international) and she is the author of more than 100 works. She is currently the dean of the "Escuela Universitaria Politécnica de Teruel". She was IEEE Senior Member (2006) and founder of the IEEE Education Society Spanish Chapter, from which she was vicechairwoman (2008-2010) and chairwoman (2010-2012). Besides, she has been founder of TAEE Association (Technology, learning and teaching of electronics), (vice-chair 2012-2015). She has been recognized in research and management (7 awards), some of them at international levels, for instance: - "IEEE Education Society Chapter Achievement Award" -2011Chairwoman of the Chapter, - "2011 Best Large Chapter Award - IEEE Region 8" - Chairwoman. Finally, she was awarded with the TAEE award to the Professional Career (2016) and the "Entreprising Woman 2018" in the University of Zaragoza.

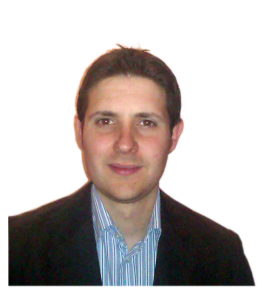

Raúl Igual (M'18) received the Ph.D. degree in Electronics Engineering from the Universidad de Zaragoza, Spain (in international modality with stays at LIRMM - Montpellier, France), in 2014, the M.S. degree in Electronics Engineering, and the B.S. degree in Telec. Engineering - Electronics Systems (with honors) in 2010 and 2008, respectively. $\mathrm{He}$ worked as associate professor at the Universidad de Zaragoza (2013-2015), and he is currently a lecturer in the Electrical Engineering Department. His main research interests include the use of sensors for quality of life applications, the evaluation of power quality in renewable energy systems, and new technologies in Higher Education. 


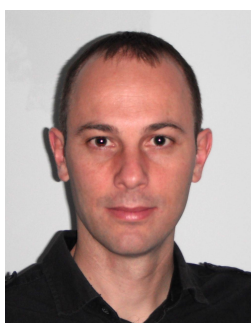

Andrés S. Lombas studied Psychology and received his Ph.D. degree in Psychology at the Basque Country University. Currently, he works as associate professor at the University of Zaragoza. Since 2009 he has been teaching Research Methods in Psychology degree at this university. His research has been mainly focused on psychological well-being, and the importance of emotional regulation, satisfaction of basic psychological needs and mindfulness to promote it.

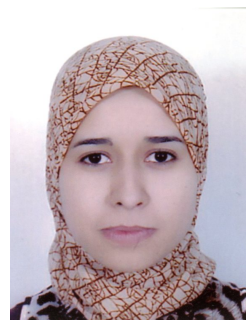

Hana Jamali is a Ph.D. student in the Cadi Ayyad University on the topic of development of an investment strategy on the stock exchange data mining approach. She has several international publications on the topic of her ongoing thesis. 